Erstes Korollar. In einem Hausdorffschen Raum ist der zweite Satz von Herrn Veress dem Durchschnittssatz von Cantor äquivalent.

Denn in einem Hausdorffschen Raum sind der Cantorsche und der Borelsche Satz miteinander äquivalent ${ }^{11}$ ).

Zweites Korollar. In dem n-dimensionalen euklidischen Raum ist der zweite Satz von Herrn Veress ohne das Auswahlaxiom. beweisbar.

Dies folgt aus dem ersten Korollar und der bekannten Tatsache, daß im n-dimensionalen euklidischen Raum der Durchschnittssatz von Gantor ohne das Auswahlaxiom beweisbar ist ${ }^{2}$ ).

Drittes Korollar. Wie wir schon oben bemerkt haben, lassen sich viele Sätze der reellen Funktionentheorie (wie z. B. die gleichmäßige Stetigkeit einer Funktion, die auf einer kompakten abgeschlossenen Menge stetig definiert ist, u. s.w.) aus dem zweiten Satz von Herrn Veress ableiten. Nach dem Obigen sind also diese Sätze im n-dimensionalen euklidischen Raum ohne das Auswahlaxiom beweisbar.

Viertes Korollar. Die gleichmäßige Stetigkeit einer Funktion, die auf einer kompakten abgeschlossenen Menge überall stetig ist, folgt aus dem Borelschen Theorem (d. h. man benötigt nicht das Borel-Lebesguesche Theorem).

Bemerkung. Es folgt aus dem Zermeloschen Axiom oder auch aus dem $V$-Postulat, daß eine Aussagenfolge, für die die Forderung (II) des ersten Satzes von Herrn Veress erfüllt ist, auf einer kompakten abgeschlossenen Menge eines Hausdorffschen Raumes auch stetig ist.

11) Dies ist ein spezieller Fall eines von Herrn S. Saks, Sur l'équivalence de deux théorèmes de la théorie des ensembles, Fund. Math. 2 (1921), p. 1-3, bewiesenen Satzes.

12) W. Sierpiński, Un théorème sur les ensembles fermés, Bull. Ac. des Sc. Cracovie (1918), p. 49-5l.

\title{
Sur les superpositions des automorphies continues d'un intervalle fermé.
}

\section{Par \\ V. Knichal (Praha).}

Soit $C$ l'ensemble de toutes les fonctions $f(x)$ croissantes et continues, définies dans l'intervalle fermé $I=\langle 0,1\rangle$ et telles que

$$
f(0)=0, \quad f(1)=1 .
$$

MiM. J. Schreier et $\mathrm{S}$. Ulam ${ }^{1}$ ) ont démontré qu'il existe cinq fonctions $\varphi_{1}, \varphi_{2}, \varphi_{3}, \varphi_{4}, \varphi_{5} \in O$ jouissant de la propriété suivante:

Quelle que soient la fonction $f_{E} C$ et $\varepsilon>0$, il existe une fonction $\varphi$ qui est une superposition finie des fonctions $\varphi_{1}, \ldots, \varphi_{5}$ et telle que

$$
|f(x)-\varphi(x)|<\varepsilon \quad \text { pour } \quad x \in I .
$$

Dans ce théorème, on peut remplacer les mots "cinq fonctions“ par les mots „deux fonctions“; on peut énoncer même le suivant

Théorème 1. Il existe deux fonctions $\varphi, \psi \epsilon O$, jouissant de la proprieté suivante:

Quelle que soient la fonction $f \in O$ et $\varepsilon>0$, il existe deus entiers positifs $n, m$ tels que

La démonstration de ce théorème sera basée sur le lemme qui suit.

1) Über topologische Abbildungen der euklidisohen Sphären, Fund. Math. 23 (1934), p. 102. 
Lemme. Il existe deux fonctions $\varphi, \psi \in C$ jouissant de la propriété suivante:

Etant donnés deux systèmes finis de nombres rationnels (à nombre égal d'éléments),

$$
\begin{aligned}
& 0<a_{1}<a_{2}<\ldots<a_{r}<1, \\
& 0<b_{1}<b_{2}<\ldots<b_{r}<1,
\end{aligned}
$$

il existe deux entiers positifs $m$ et $n$ tels que

$$
a_{i}=\psi^{n} \varphi^{m}\left(b_{i}\right) \quad \text { pour } \quad i=1,2, \ldots, r .
$$

Démonstration. On dira que la fonction $\chi \epsilon C$ jouit de la propriété $(P)$, si $\chi(x)>x$, pour $0<x<1$. On a

$$
\lim _{n \rightarrow \infty} \chi^{n}(x)=1
$$

pour $0<x<1$ et pour toutes les fonctions $\chi(x)$ jouissant de la propriété $(P)$. En effet, la suite $\chi^{n}(x)$ est croissante, donc convergente. Si l'an avait $0<\lim \chi^{n}(x)=\beta<1$, on aurait aussi

$$
\chi(\beta)=\chi\left(\lim \chi^{n}(x)\right)=\lim \chi^{n+1}(x)=\beta,
$$

ce qui est en contradiction avec la propriété $(P)$ de $\chi(x)$.

Posons $\varphi(x)=x^{2}$ pour $x \in I$ et soit $\left\{C^{i}\right\}$ la suite de tous les systèmes finis de nombres rationnels choisis dans l'intervalle ouvert $(0,1)$. Ordonnons en une suite tous les couples $\left(C^{r}, C^{s}\right)$, où $r, s$ sont des entiers positifs et $C^{r}, C^{s}$ contiennent le même nombre d'élements:

$$
\left(A^{1}, B^{1}\right),\left(A^{2}, B^{2}\right),\left(A^{3}, B^{3}\right), \ldots
$$

Nous allons construire par induction une suite $\left\{\alpha_{i}\right\}$ de nombres réels, une suite $\left\{\psi_{j}\right\}$ de fonctions de $C$ et deux suites $\left\{n_{i}\right\},\left\{m_{i}\right\}$ de nombres entiers positifs qui satisfont aux conditions:

$$
\begin{array}{lc|}
\text { 1. } & a_{0}>a_{1}>a_{2}>\ldots>0, \\
2 . & a_{i} \leqslant 1 / 2^{i+1} \\
\left.3^{2}\right) . & a_{i}<A^{i+1} \\
4 . & \psi_{i} \text { jouit de la propriété }(P) \mid \text { pour } i \\
5 . & \psi_{i+1}(x)=\psi_{i}(x) \text { pour } \alpha_{i} \leqslant x \leqslant 1 \\
\left.6^{2}\right) . & \varphi^{m_{i}}\left(B^{i}\right)>\alpha_{i} \\
7 . & \psi_{i}^{n_{l}} \varphi^{m_{i}}\left(B^{i}\right)=A^{i}
\end{array}
$$

2) $A, B$ étant deux systèmes de nombres réels, $A<B$ signifie que les conditions $a \in A$ et $b \in B$ entraînent $a<b$. La signification de $a<B$ est tout à fait analogue.

Posons (conformément aux conditions 1-7) $\quad \psi_{0}(x)=\sqrt{\grave{x}}$ et choisissons un $\alpha_{0}$ tel qu'on ait $0<\alpha_{0} \leqslant \frac{1}{2}$ et $a_{0}<A^{1}$. Les éléments:

$$
\alpha_{0}, \ldots, \alpha_{i}, \quad \psi_{0}, \ldots, \psi_{i}, \quad n_{1}, \ldots, n_{i}, \quad m_{1}, \ldots, m_{i}
$$

étant choisis conformément aux conditions 1-7, définissons

comme il suit.

$$
\alpha_{i+1}, \quad \psi_{i+1}, \quad n_{l+1}, \quad m_{i+1}
$$

Soit $m_{i+1}$ un entier positif et suffisamment grand pour qu'on ait

$$
0<D^{i+1}=\varphi^{m_{i+1}}\left(B^{i+1}\right)<\alpha_{i} .
$$

Choisissons un $\alpha_{i+1}$ tel qu'on ait

$$
0<\alpha_{i+1}<\operatorname{Min}\left(1 / 2^{i+2}, A^{i+2}, D^{i+1}\right) .
$$

et un entier positif $n_{i+1}$ tel qu'on ait (voir (3))

$$
\beta=\psi_{i}^{n_{i+1}}\left(\alpha_{i}\right)>A^{i+1} \text {. }
$$

On a d'après (6), (5), la condition 3 pour $\alpha_{i}$ et (7)

$$
0<\alpha_{i+1}<D^{i+1}<\alpha_{i}<A^{i+1}<\beta<1 .
$$

Il existe évidemment (voir (7) et (8)) une fonction $\chi \in C$ telle que:

$$
\begin{gathered}
\chi\left(D^{i+1}\right)=A^{i+1}, \\
\chi(x)=\psi_{i}^{n_{i+1}}(x) \quad \text { pour } \quad a_{i} \leqslant x \leqslant 1
\end{gathered}
$$

(les systèmes $D^{i+1}$ et $A^{i+1}$ contenant le même nombre d'éléments). Posons enfin ${ }^{3}$ )

$$
\psi_{i+1}(x)=\chi^{-1} \psi_{i} \chi(x) \quad \text { pour } \quad 0 \leqslant x \leqslant 1 .
$$

On a évidemment $\psi_{i+1} \epsilon C$. Il faut démontrer que les conditions 1-7 sont valables aussi pour les éléments $m_{i+1}, a_{i+1}, n_{i+1}, \psi_{i+1}$. Les conditions 1-3 le sont évidemment (voir (6)). On a $\psi_{i} \chi(x)>\chi(x)$ pour $0<x<1$, car $\psi_{i}$ jouit de la propriété $(P)$. et $0<\chi(x)<1$. La fonction $\chi^{-1}(x)$ étant croissante, on a

$$
\chi^{-1} \psi_{l} \chi(x)>\chi^{-1} \chi(x)=x \quad \text { pour } 0<x<1
$$

a) $\chi^{-1}$ signifie la fonction inverse par rapport à $\chi$. Fundamenta Mathematicae. T. Xxxt. 
La fonction $\psi_{i+1}$ satisfait donc à la condition 4 , d'où

$$
\alpha_{i} \leqslant \psi_{i+1}(x) \leqslant 1 \quad \text { pour } \alpha_{i} \leqslant x \leqslant 1,
$$

done selon (10) et (11) pour $\alpha_{i} \leqslant x \leqslant 1$ :

$$
\begin{array}{ll}
\text { tantôt } & \psi_{i} \chi(x)=\psi_{i} \psi_{i}^{n_{i+1}}(x)=\psi_{i}^{1+n_{i+1}}(x), \\
\text { tantôt } & \psi_{i} \chi(x)=\chi \psi_{i+1}(x)=\psi_{i}^{n_{i+1}} \psi_{i+1}(x),
\end{array}
$$

de sorte que l'on a $\psi_{i}(x)=\psi_{i+1}(x)$ en accord avec la condition 5 .

La condition 6 est évidemment satisfaite d'après (5) et $(6)$. Enfin, on a d'après (11), (9), (8) et (10)

$$
\psi_{i+1}^{n_{i+1}}\left(D^{i+1}\right)=\chi^{-1} \psi_{i}^{n_{i+1}} \chi\left(D^{i+1}\right)=\chi^{-1} \psi_{i}^{n_{i+1}}\left(A^{i+1}\right)=\chi^{-1} \chi\left(A^{i+1}\right)=A^{i+1},
$$

c. à d. la condition 7 .

La suite $\psi_{0}(x), \psi_{1}(x), \psi_{2}(x), \ldots$ est, d'après les conditions 1,2 et 5 convergente pour $0 \leqslant x \leqslant 1$. Posons donc

On a évidemment

$$
\psi(x)=\lim _{i \rightarrow \infty} \psi_{i}(x) .
$$

$$
\psi(x)=\psi_{i}(x) \quad \text { pour } \quad \alpha_{i} \leqslant x \leqslant 1 .
$$

Les conditions $7,6,(12)$ et la propriété $(P)$ de $\varphi_{i}^{?}$ entraînent

$$
A^{i}=\psi_{i}^{n_{i}} \varphi^{m_{i}}\left(B^{i}\right)=\psi^{n_{i}} \varphi^{m_{i}}\left(B^{i}\right) .
$$

Il est facile de voir que $\psi \epsilon C$. En effet, la continuité de la fonction $\psi(x)$ au point $x=0$ résulte immédiatement de (13) et du fait qu'il existe pour tout $\varepsilon>0$ un nombre naturel $i$ tel que $A^{i}<\varepsilon$.

Démonstration du théorème 1. Soient $f \epsilon O, \varepsilon>0$ et $r$ un entier positif tel que:

$$
0 \leqslant x \leqslant 1, \quad 0 \leqslant x^{\prime} \leqslant 1, \quad\left|x-x^{\prime}\right| \leqslant 1 / r
$$

entraîne $\left|f(x)-f\left(x^{\prime}\right)\right|<\varepsilon / 4$.

Posons $b_{i}=i / r$ pour $i=0,1,2, \ldots, r$ et $b_{-1}=0$, et choisissons les nombres rationels $a_{i}$ de manière qu'on ait

$$
0=a_{0}=f\left(b_{0}\right)<a_{1}<f\left(b_{1}\right)<a_{2}<f\left(b_{2}\right)<\ldots<a_{r}=f\left(b_{r}\right)=1 .
$$

Il existe en vertu du lemme deux entiers positifs $n$ et $m$ tels que l'on a, en posant $\chi(x)=\psi^{n} p^{m}(x)$,

$$
\chi\left(b_{i}\right)=a_{i} \quad \text { pour } \quad i=0,1, \ldots, r .
$$

Alors, pour $b_{i-1} \leqslant x \leqslant b_{i}$ (où $i=1,2, \ldots, r$ ), on peut raisonner comme il suit (voir (14), (15) et (16)):

$$
\begin{gathered}
\left|f(x)-f\left(b_{i}\right)\right|<\varepsilon / 4, \\
\left|f\left(b_{i}\right)-\chi\left(b_{i}\right)\right|=\left|f\left(b_{i}\right)-a_{i}\right| \leqslant\left|f\left(b_{i}\right)-f\left(b_{i-1}\right)\right|<\varepsilon / 4, \\
\left|\chi\left(b_{i}\right)-\chi(x)\right| \leqslant\left|a_{i}-a_{i-1}\right| \leqslant\left|f\left(b_{i}^{*}\right)-f\left(b_{i-2}\right)\right|<2 \varepsilon / 4,
\end{gathered}
$$

donc $|f(x)-\chi(x)|<\varepsilon$, c. q.f. d.

Théorème 2. Dans le théorème 1, on ne peut pas remplacer les mots "deux fonctions" par les mots "une fonction".

Pour la démonstration, il suffit de remarquer que la suite $\left\{p^{n}(x)\right\}$ est convergente, quel que soient $x \in I$ et $\varphi \epsilon C$. 